\title{
Correlative Electron and Fluorescence Microscopy of Magnetotactic Bacteria in Liquid: Toward In Vivo Imaging
}

Taylor J. Woehl ${ }^{1}$, Sanjay Kashyap ${ }^{1}$, Emre Firlar ${ }^{1}$, Teresa Perez-Gonzalez ${ }^{2}$, Damien Faivre ${ }^{2}$, Denis Trubitsyn ${ }^{3}$, Dennis A. Bazylinski ${ }^{3}$, and Tanya Prozorov ${ }^{1}$

${ }^{1}$ Emergent Atomic and Magnetic Structures, Division of Materials Sciences and Engineering, Ames Laboratory, Ames, IA 50011, USA.

2 Department of Biomaterials, Max Planck Institute of Colloids and Interfaces, Science Park Golm, 14424 Potsdam, Germany.

${ }^{3}$ School of Life Sciences, University of Nevada at Las Vegas, Las Vegas, NV 89154, USA.

Magnetotactic bacteria, present in many natural aquatic environments, biomineralize ordered chains of uniform magnetite or greigite nanocrystals, also known as magnetosomes [1]. These nanoparticles exhibit nearly perfect crystal structures, faceting, and consistent species-specific morphologies, leading to well-defined magnetic properties. As a result, magnetotactic bacteria can serve as a model system for the study of the molecular mechanisms for magnetite biomineralization [2]. However, little is known about their complex formation mechanism. Transmission electron microscopy (TEM) can provide critical information about the organization of the magnetosomes and the growth mechanisms by revealing the nanoparticle structure on the atomic level [3]. Conventional TEM traditionally does not allow imaging in native liquid or atmospheric environments because of the high vacuum of the specimen enclosure. The vitreous ice environment used during cryo-TEM imaging of bacteria [4] is not entirely representative of the native hydrated cellular state either, and may introduce artifacts due to specimen preparation and electron radiation damage [5]. Therefore direct observation of the time-dependent growth of magnetosomes in their native cellular environment remains a significant challenge.

Here we utilize correlative fluorescence and fluid cell scanning TEM (STEM) imaging to visualize magnetotactic bacteria containing nanoscale biomineralized magnetosomes [6]. Fluorescently labeled cells of Magnetospirillum magneticum strain AMB-1 were immobilized on microchip window surfaces and visualized in a fluid cell with high angle annular dark field (HAADF) STEM, followed by correlative fluorescence imaging (Figure 1). The confined environment of the fluid cell caused a rapid decrease in bacteria viability over a time of two hours, necessitating the use of post-STEM fluorescence microscopy to verify bacteria membrane integrity. Notably, the post-STEM fluorescence imaging indicated that the bacterial cell wall membrane did not sustain radiation damage during STEM imaging at low electron dose conditions $\left(<0.2\right.$ electrons $\left.\cdot \AA^{2}\right)$. Correlative STEM and fluorescence imaging of magnetotactic bacteria is a first step in observing biomineralization of magnetite nanocrystals in vivo, and the described approach is expected to be applicable to a broad range of microorganisms that biomineralize various nanomaterials [7]. 
References:

[1] D.A. Bazylinski, R.B. Frankel, Nature Reviews Microbiology, 2 (2004) 217-230.

[2] D. Faivre, D. Schüler, Chem. Rev., 108 (2008) 4875-4898.

[3] R.E. Dunin-Borkowski, M.R. McCartney, R.B. Frankel, D.A. Bazylinski, M. Posfai, P.R. Boseck, Science, 282 (1998) 1868-1870.

[4] A. Komeili, H. Vali, T.J. Beveridge, D.K. Newman, Proceeding of the National Academy of Sciences of the United States of America, 101 (2004) 3839-3844.

[5] J. Dubochet, M. Adrian, J.J. Chang, J.C. Homo, J. Lepault, A.W. McDowall, P. Schultz, Quarterly Review of Biophysics, 21 (1988) 129-228.

[6] T.J. Woehl, S. Kashyap, E. Firlar, T. Perez-Gonzalez, D. Faivre, D. Trubitsyn, D.A. Bazylinski, T. Prozorov, Scientific Reports, 4 (2014).

[7] This work was supported by the U.S. Department of Energy, Office of Basic Energy Science, Division of Materials Sciences and Engineering. The research was performed at the Ames Laboratory, which is operated for the U.S. Department of Energy by Iowa State University under Contract No. DE-AC02-07CH11358. T.P. acknowledges support from the Department of Energy Office of Science Early Career Research Award, Biomolecular Materials Program. The authors would like to thank C. L. Mosher, M. J. Kramer, and T. M. Pepper for informative discussions. This research was supported in D.F's laboratory by the Max Planck Society, and a starting grant from the ERC (Project MB2, no. 256915).
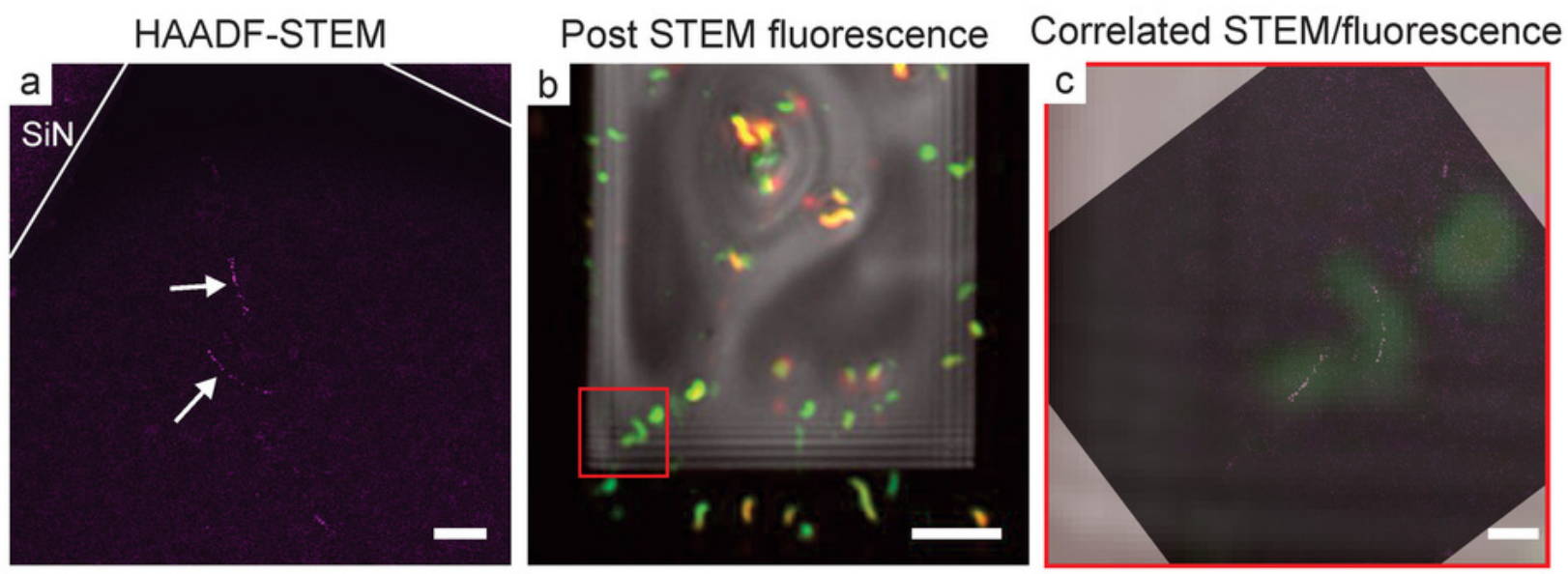

Figure 1. Correlated liquid cell STEM and fluorescence images of magnetotactic bacteria with intact membranes. (a) False colored, background subtracted HAADF-STEM image of two bacterial cells near the corner of the SiN window, the magnetosome chains appear in purple and are denoted with white arrows. (b) Post-STEM composite fluorescence image of the same fluid cell sample. (c) Correlated STEM and composite fluorescence image of the bacterial cells highlighted in the red box in (b). The scale bar is $1 \mu \mathrm{m}$ in (a) and (c) and $10 \mu \mathrm{m}$ in (b). 